\title{
Guide to Introduction to Everyday Clinical Practice
}

\author{
Ole Ackermann
}

The introduction of new methods into everyday clinical practice is always a challenge. Whether this is planned in a practice or clinic, the problems are similar. Colleagues who are used to X-ray findings must be convinced, dealing with new processes must be practiced and the diagnostic method itself trained. Complete, safe, and fast treatment of patients must also be guaranteed during the changeover phase.

Always have in mind: safety first! Whenever there is any doubt about the finding, an X-ray image is permitted and useful. Uncertainties always occur, especially in the initial phases, and it is best to eliminate them immediately and consistently. Nothing is more deadly for a new method than a misdiagnosis and many a hopeful start has failed because of it. Therefore, it is necessary to make the beginning safe for doctors and patients, with increasing routine the need for $\mathrm{X}$-ray checks will be quickly minimized.

\subsection{The Doctor Starts}

To gain security as a beginner in sonographic fracture diagnostics, it is essential to first familiarize yourself with the possibilities and limits of the procedure. One has to realize that ultrasound always displays the surface of the bone. In the beginning, there are also often concerns that the examination could be painful. We can deny this from the experience of several thousand investigations.

The start of diagnostics with the distal fracture of the forearm is an ideal beginning. The fracture is often easy to visualize and, due to the high correction potential, very safe in therapy. In the beginning, it makes sense to carry out the sonographic examination, then commit yourself to a diagnosis and treatment, and then carry out an X-ray examination. The examiner will then see that the wrist SAFE leads to the goal. After 10-20 examinations, the necessary security is

O. Ackermann $(\bowtie)$

Department of Orthopedic Surgery, Ruhr-University Bochum, Bochum, Germany usually available to use the X-ray examination only in exceptional cases.

Based on this basic knowledge, ultrasound diagnostics can then be extended to other areas.

Of course, a practical course to introduce the methodology is helpful.

\subsection{Introduction to Practice}

Experience has shown that the main obstacle is the availability of the (often the only) ultrasound device. The processes should be structured so that patients with suspected fractures are generally examined in the ultrasound room. Only then will the speed advantage of sonography become noticeable.

Even if the vast majority of parents prefer ultrasound imaging to X-ray diagnostics, it always happens that an $\mathrm{X}$-ray examination is desired. In our experience, there is no point in discussing at this point, otherwise, the X-ray will be made up elsewhere. We proceed in such a way that we then carry out an ultrasound examination and also an X-ray image.

\subsection{Introduction to the Clinic}

If the clinic chief has not learned the method himself and wants to introduce it, there are several hurdles to be overcome. Every employee must be aware that the chief doctor is responsible for the clinic and, in case of doubt, is personally responsible for every procedure and every mistake. Therefore, he will only introduce a new method if he is convinced of its quality. To achieve this, it makes sense to first run ultrasound and X-ray diagnostics consistently in parallel and to present both in the X-ray discussion. Employees should also be free to use the technology in order to achieve good results.

If the method has proven its potential over time, a standard application can be discussed. 